Cahiers Charlevoix

Études franco-ontariennes
Cahiers Charlevoix Études franco-ontariennes

or Crevenerix of

\title{
Études universitaires en français en Ontario : entre motivations personnelles et contraintes institutionnelles
}

\section{Julie Boissonneault}

Volume 11, 2016

URI : https://id.erudit.org/iderudit/1039285ar

DOI : https://doi.org/10.7202/1039285ar

Aller au sommaire du numéro

Éditeur(s)

Société Charlevoix

Presses de l’Université d’Ottawa

ISSN

1203-4371 (imprimé)

2371-6878 (numérique)

Découvrir la revue

Citer cet article

Boissonneault, J. (2016). Études universitaires en français en Ontario : entre motivations personnelles et contraintes institutionnelles. Cahiers Charlevoix, 11, 157-191. https://doi.org/10.7202/1039285ar
Résumé de l'article

L'étude de Julie Boissonneault s'inscrit dans le droit fil de ses préoccupations depuis plus d'une décennie et en parfait synchronisme avec les revendications contemporaines des étudiants francophones, spécialement celles de 2014 et 2015, pour le droit à l'université de langue française en Ontario. Portant son regard sur la fréquentation des établissements universitaires, elle se demande dans quelle langue les Franco-Ontariens font leur scolarité. Sont alors mises en jeu la langue maternelle et la langue de la scolarité à l'élémentaire et au secondaire comme vecteur de leur motivation à étudier en français au palier supérieur. Pour mener à terme son projet, elle analyse le discours même d'un groupe d'étudiants inscrits à l'Université Laurentienne en 2005, obtenu à l'aide d'un questionnaire par lequel ils expriment leurs motivations. L'article lève ainsi le voile sur une facette peu explorée dans le choix d'étudier dans la langue de la minorité : celle des contraintes institutionnelles qui entravent l'intention et la réalisation des études universitaires en français. 


\section{Études universitaires en français en Ontario : entre motivations personnelles et contraintes institutionnelles}

Julie BoissonneAult

Département d'études françaises

Université Laurentienne 


\section{SOMMAIRE}

INTRODUCTION

I - FrancophONIE MINORITAIRE AU POSTSECONDAIRE 162

II - Choix de la langue D'enseignement À L'Université 168

III - Que Disent Les ÉtUdiants ? $\quad 172$

1. Profil des répondants 173

2. Choix de la langue de la scolarité à l'université 175

3. Justification du choix de la langue d'instruction 178

a) Francophones qui étudient uniquement en français

178

b) Francophones qui étudient plutôt en français

181

c) Francophones qui étudient autant en français qu'en anglais 183

d) Francophones qui étudient en anglais $\quad 184$

e) Étudiants anglophones 185

IV - QUE SE DÉGAGE-T-IL DE LEUR DISCOURS ? 187

$\begin{array}{ll}\text { CONCLUSION } & 189\end{array}$ 


\section{Études universitaires en français en Ontario : entre motivations personnelles et contraintes institutionnelles}

\section{INTRODUCTION}

Le nombre de Canadiens qui font des études postsecondaires, surtout des études de premier cycle universitaire, a augmenté considérablement depuis les vingt dernières années. Une étude longitudinale menée par l'Organisation de coopération et développement économiques sur l'état du parcours de jeunes étudiants canadiens de 1995-1996 à 2006-2007 indique que le taux d'inscription aux programmes de premier cycle universitaire a connu une hausse de $40 \%$ pendant cette décennie et que le nombre de diplômés a augmenté de $21 \%{ }^{1}$. Cette hausse est attribuable à l'importance croissante qu'accordent les sociétés occidentales aux études postsecondaires et au fait que « l'université est devenue une porte d'accès à la vie active de plus en plus sollicitée ${ }^{2}$ ».

Or, choisir de faire des études postsecondaires n'est pas une sinécure. Le jeune adulte qui décide d'étendre sa formation audelà du secondaire se pose de nombreuses questions. Il lui faut d'abord découvrir ses intérêts, ses compétences et ses visées professionnelles afin de déterminer le domaine d'études dans lequel s'investir. Puis, il lui faut tenir compte de facteurs divers quant au choix de l'établissement à fréquenter, selon les programmes d'études, la proximité géographique de l'institution et les coûts

1. Organisation de coopération et développement économiques (OCDE), Les Clés de la réussite. Impact des connaissances et compétences à l'âge de 15 ans sur le parcours de jeunes Canadiens, OCDE, 2010, www.oecd.org/pisa/44576 (consulté le 7 novembre 2014).

2. Maud Lemaire et Cameron Montgomery, «Exogamie et choix de la langue d'enseignement au niveau universitaire au Canada : six études de cas », DiversCité Langues, vol. VIII, 2003, www.teluq.uqebec.ca/diverscite (consulté le 11 janvier 2015). 
(scolarité, logement) qui s'y rattachent. Selon le Conseil canadien sur l'apprentissage, les jeunes Canadiens choisissent de faire ou non des études postsecondaires (collégiales ou universitaires) et d'y persévérer selon les facteurs que sont «l'attrait de l'emploi au sein d'une économie forte » et la présence d'obstacles : situation financière, attitudes des parents à l'égard des études postsecondaires, rendement scolaire, engagement social et scolaire au secondaire, compétences en lecture, en sciences et en mathématiques et proximité d'un établissement postsecondaire ${ }^{3}$.

Les francophones canadiens vivant en milieu minoritaire sont confrontés à une autre question d'importance, celle de la langue de la scolarité. Étudieront-ils en français ou choisiront-ils de le faire en anglais ? Bien qu'elle ne soit pas propre aux seuls francophones, cette question revêt une importance différente selon qu'elle se pose pour des locuteurs en situation minoritaire ou pour des locuteurs dont la langue première est celle de la majorité. Les anglophones, en l'occurrence, ont également la possibilité de faire leurs études en français et peuvent, à ce titre, se poser la question, mais les enjeux sociaux et individuels des uns et des autres ne sont pas équivalents, bien qu'il s'agisse des deux mêmes langues ${ }^{4}$. La situation diglossique dans laquelle coexistent les deux langues (situation qui varie selon les régions et le poids démographique) touche à la fois l'usage que l'on fait du français et de l'anglais et l'importance qu'on attribue à chacune de ces langues. Un usage moindre et une importance faible entraînent souvent, auprès des locuteurs de la langue minoritaire, des sentiments d'insécurité linguistique qui, par ricochet, peuvent mener à l'acculturation ${ }^{5}$.

3. Conseil canadien sur l'apprentissage, L'Enseignement postsecondaire au Canada : les attentes sont-elles comblées? Ottawa, 2009, 161 p., www.ccl.cca.ca (consulté le $1^{\text {er }}$ mars 2009).

4. Des nombreuses minorités linguistiques au Canada, deux seules sont de langue officielle : celle de langue française et celle de langue anglaise. Il s'agit des deux seules langues dans lesquelles toute instruction de l'ordre public est dispensée au pays.

5. Voir Tina Desabrais, « Les mots pour le dire... L'influence de l'(in)sécurité linguistique sur l'expérience d'étudiantes de milieux francophones minoritaires canadiens inscrites aux études supérieures à l'Université d'Ottawa ", Thèse doctorale, 
On ne choisit ainsi généralement pas d'étudier dans la langue de la minorité pour les mêmes motifs selon qu'il s'agisse de sa langue première $\left(\mathrm{L}_{1}\right)^{6}$ ou de sa langue seconde $\left(\mathrm{L}_{2}\right)$.

C'est précisément au choix de la langue de scolarité à l'université effectué par les francophones de l'Ontario que nous nous intéressons. Le but n'est pas tant de déterminer le nombre d'individus qui choisissent de se former dans une langue ou dans une autre que de tenter de mieux tisser la complexité des motivations sous-jacentes aux choix que font les Franco-Ontariens de s'inscrire à un programme de langue française ou de langue anglaise et d'y faire leur formation. Ces motivations sont-elles de l'ordre de l'utilitaire et du pragmatisme (accessibilité à des programmes d'études et disponibilité des programmes) ou relèvent-elles de l'ordre culturel et idéologique (représentation linguistique personnelle ou collective) ? Quelles attitudes, croyances, valeurs (esthétiques, morales et affectives) et normes implicites ou explicites à l'égard de la langue animeront leurs discours ? Poseront-ils un jugement d'ordre métalinguistique (facilité ou difficulté inhérente attribuée à la langue), un jugement sur la communauté linguistique dans laquelle s'inscrit la langue (utilité et vitalité sociales) ou un jugement sur eux-mêmes en tant que locuteurs francophones (compétence langagière et sens d'appartenance)?

Afin d'y répondre, nous dresserons d'abord le portrait de la francophonie canadienne en matière d'éducation postsecondaire, et poserons la problématique en nous inspirant d'études menées auprès de Franco-Canadiens en milieu minoritaire - notamment auprès des francophones de l'Ontario. Puis, nous analyserons ce qu'en disent des étudiants, à partir de données recueillies en 2005 à l'Université Laurentienne. Sera ainsi juxtaposé le choix de la langue de la formation universitaire aux facteurs qui agissent sur ce choix à partir des raisons que donnent les étudiants de faire ou non leur scolarité en français.

Faculté d'éducation et Programme pluridisciplinaire de doctorat en études canadiennes, Université d'Ottawa, 2013.

6. Entendu ici comme langue maternelle et première langue apprise à la maison. 


\section{I - FranCOPHONIE MINORITAIRE AU POSTSECONDAIRE}

Que savons-nous de la formation postsecondaire des FrancoCanadiens, plus précisément des Franco-Ontariens ? Peu de choses, en fait. Le recensement canadien de 2011, qui brosse un portrait succinct de la situation, indique que près des deux tiers (64,1\%) des Canadiens âgés de 25 à 64 ans avaient terminé des études postsecondaires en 20117. L'Enquête nationale auprès des ménages (ENM) de 2011 chiffre ce taux à plus de onze millions d'individus (11 782700$)^{8}$. Ces individus n'ont pas tous fréquenté l'université, d'autres formations postsecondaires étant à leur portée (collège, école de formation professionnelle ou de métiers) ${ }^{9}$. Selon les données du dernier recensement, un peu plus de trois millions (3 032 220) d'individus avaient terminé une formation universitaire, de premier cycle ou de cycles supérieurs, ce qui représente $16,5 \%$ de la population canadienne âgée de 25 à 64 ans $^{10}$.

C'est en Ontario qu'on trouvait, en 2011, la plus forte proportion de la population adulte, âgée de 25 à 64 ans, à détenir un grade universitaire : $28,9 \%{ }^{11}$, ce qui ne surprend pas outre mesure, puisque la province compte, à elle seule, plus du tiers de la population canadienne avec ses quelque douze millions d'habitants ${ }^{12}$.

7. Statistique Canada, «Indicateurs de l'éducation au Canada : une perspective internationale, 2013 », Le Quotidien, le 7 janvier 2014, $\mathrm{n}^{\circ} 11-001-\mathrm{X}$ au catalogue de Statistique Canada, www12.statcan.gc.ca/census-recensement/2011 (consulté le 6 novembre 2014).

8. Statistique Canada, Scolarité au Canada : niveau de scolarité, domaine d'études et lieu des études, Enquête nationale auprès des ménages, 2011, Ottawa, Ministre de 1'Industrie, 2013, no 99-012-X2011001 au catalogue, www.statcan.gc.ca (consulté le 6 novembre 2014).

9. En fait, $37 \%$ des Canadiens détiendraient un titre inférieur au baccalauréat universitaire. Ceci est en partie le reflet du vaste réseau de collèges au Canada, lesquels sont beaucoup moins répandus dans la plupart des autres pays de l'OCDE [Organisation de coopération et de développement économiques] (Statistique Canada, « Indicateurs de l'éducation au Canada... », op. cit.).

10. Statistique Canada, Scolarité au Canada ... op. cit.

11. Ibid.

12. En 2011, la population canadienne se chiffrait à 33121175 d'habitants, et celle de l'Ontario à 12722 065. Voir Statistique Canada, Population selon la langue maternelle et les groupes d'âge (total), pour le Canada, les provinces et les 
Ce portrait, qui souligne l'importance de la formation postsecondaire au Canada, demeure incomplet puisqu'il ne fournit pas la distribution en fonction de la langue première ${ }^{13}$ des individus et n'indique pas la langue dans laquelle se fait la scolarité. En fait, de nombreuses études attestent toujours de la disparité qui sévit, au Canada, entre les francophones et les anglophones au chapitre des études postsecondaires, et ce, même si la scolarité s'allonge de plus en plus chez les premiers. Étudier en français « de la petite enfance jusqu'au postsecondaire, continue à représenter un défi de taille pour chacune des communautés francophones et acadienne ${ }^{14} \gg$.

Dans un rapport sur l'éducation en Ontario, l'ancien premier ministre, Bob (Robert) Rae, reconnaissait, en 2005, cette disparité entre les locuteurs de langue minoritaire et ceux de langue majoritaire :

Au sein de cette province canadienne à majorité anglophone, l'accès aux études postsecondaires et aux échelons économiques et politiques supérieurs de la société se caractérise en effet par une grande disparité historique entre anglophones et francophones (Allaire et Toulouse, 1973 ; Mougeon et Heller, 1986 ; Welch, 1993). Malgré l'obtention de plusieurs acquis - au prix de luttes sociales et politiques acharnées - les jeunes francophones de l'Ontario font, encore aujourd'hui, partie des catégories étudiantes qui rencontrent le plus d'obstacles à leur accès aux études supérieures ${ }^{15}$.

Cette disparité s'explique par l'histoire truffée d'embûches et de crises de l'éducation en langue française en situation minoritaire.

territoires, 22 août 2014, www12.statcan.ca/census-recensement/2011 (consulté le 6 novembre 2014).

13. L'analyse en fonction de la langue première des individus tient compte de la langue maternelle ainsi que de la langue d'usage en milieu familial et en milieu institutionnel.

14. Réal Allard, Rodrigue Landry et Kenneth Deveau, Et après le secondaire? Étude pancanadienne des aspirations éducationnelles et intentions de faire carrière dans leur communauté des élèves de $12^{e}$ année d'écoles de langue française en situation minoritaire, Institut canadien de recherche sur les minorités linguistiques, 2009, p. 13, www.qspace.library.queensu.ca (consulté le 7 novembre 2014).

15. Robert Rae, L'Ontario : chef de file en éducation. Rapport et recommandations, Gouvernement de l'Ontario, Imprimeur pour la Reine, 2005, p. 6. 
Bien que les gains en matière d'éducation en langue française aient commencé à se voir dès 1968, ils n'ont pris de l'essor que dans les années 1990. On se souviendra que ce n'est qu'en 1968 (un an avant l'adoption de la première Loi sur les langues officielles du Canada), que sera mis en place un réseau d'écoles secondaires publiques (par la loi 141) ${ }^{16}$. S'ensuivra une légitimation de l'éducation dans la langue de la minorité de langue officielle, en 1982, avec la promulgation de l'article 23 de la Charte canadienne des droits et libertés qui donnera officiellement accès, à l'échelle nationale, à l'éducation dans la langue de la minorité officielle, mais aux seuls paliers élémentaire et secondaire. Se produira alors une augmentation de la scolarité en langue française au pays - tous niveaux confondus -, tant auprès des locuteurs de langue maternelle qu'auprès des individus ayant le français comme langue seconde. En 1986, il y aura parachèvement provincial, c'est-à-dire que les écoles confessionnelles (catholiques) seront financées à même les fonds publics et, en 1997, les francophones obtiendront la pleine gestion scolaire : les conseils scolaires autonomes de langue française verront alors le jour.

Malgré ces acquis, les francophones de l'Ontario ne disposent que de peu de choix s'ils désirent faire des études postsecondaires en français. Ils peuvent se tourner vers le collégial, puisque, en 1990, a été créé le premier collège de langue française à Ottawa - la Cité collégiale - et, cinq ans plus tard, le collège Boréal à Sudbury et le collège des Grands Lacs à Toronto. La province n'a cependant aucun établissement autonome de langue française au niveau universitaire ${ }^{17}$. L'Ontario abrite, pourtant, la plus grande communauté de langue française en situation minoritaire au pays, soit 493300 locuteurs dont la seule langue maternelle est le français, ce qui représente $3,9 \%$ de la population ontarienne

16. Amendement de 1968 à la Loi sur les écoles secondaires et les conseils scolaires. Voir Centre de recherches en civilisation canadienne-française (CRCCF), La Présence française en Ontario : 1610, passeport pour 2010, Université d'Ottawa, 2003, www.crccf.uottawa.ca (consulté le 9 mars 2015).

17. Voir Serge Dupuis, Alyssa Jutras-Stewart et Renée Stutt, « L'Ontario français et les universités bilingues (1960-2015) », Revue du Nouvel-Ontario, n ${ }^{\circ}$, 2015, p. 13-104, www.erudit.org. 
(selon les données du recensement canadien de 2011 ${ }^{18}$ ) ou 611500 individus représentant $4,8 \%$ de la population provinciale si l'on adhère à la Définition inclusive des francophones (DIF) mise de l'avant par l'Ontario en $2009^{19}$.

La province compte aussi, à elle seule, 19 universités et 24 collèges communautaires. De prime abord, ce nombre élevé d'établissements pourrait constituer un élément qui faciliterait les études en français. Toutefois, si l'on désire étudier en français, les choix sont beaucoup moins élevés. Sur les 24 collèges d'arts appliqués et de technologie de l'Ontario, deux seuls - le collège Boréal (Sudbury et Toronto ${ }^{20}$ ) et la Cité collégiale (Ottawa) ont des programmes d'études uniquement en langue française. Aucune des universités autonomes (c'est-à-dire conférant un grade) n'est de langue française. Deux d'entre elles sont désignées comme étant bilingues (anglais-français) et ont certains programmes en français : d'une part, l'Université Laurentienne (à laquelle 1'Université de Hearst est affiliée et 1'Université de Sudbury, fédérée), et, d'autre part, l'Université d'Ottawa (l'Université Saint-Paul y étant fédérée) ${ }^{21}$. L'Université York (Toronto) a un campus bilingue (le Collège Glendon), l’Université de Guelph a des programmes de formation agricole et horticole en langue française par son affiliation au collège d'Alfred, l'Université de Toronto, des programmes en éducation en langue française par

18. Ces données ne comprennent pas les 67860 Ontariens qui ont déclaré avoir le français ainsi qu'une autre langue comme langue maternelle (Statistique Canada, Population selon la langue maternelle... op. cit.).

19. Ontario, Office des affaires francophones (5 juin 2014), www.ofa.gov. on.ca. Selon la Dif, sont «francophones » non seulement les individus qui ont le français comme langue(s) maternelle(s), mais également ceux qui n'ont ni le français, ni l'anglais comme langue maternelle, mais qui ont une bonne connaissance du français et qui l'utilisent à la maison. Cette définition comprend ainsi les locuteurs pour qui le français est la première langue d'usage sans en être la langue maternelle, ce qui permet d'inclure les nouveaux arrivants.

20. Créé en 1995, le collège des Grands Lacs fermera ses portes en 2001. La formation collégiale en langue française pour la région du Centre-Sud sera alors attribuée au collège Boréal.

21. Voir Gratien Allaire, «Unilinguisme, bilinguisme et institution universitaire dans la francophonie des années 1960 », Francophonies d'Amérique, n 14, 2002, p. 101-116, www.erudit.org (consulté le 17 novembre 2014). 
le truchement de l'Institut d'études pédagogiques de l'Ontario, et l'Université Carleton, des programmes de philosophie et de théologie en français au Collège universitaire dominicain.

Seules quelques études empiriques documentent les études postsecondaires des francophones de l'Ontario. Stacy Churchill, Normand Frenette et Saeed Quazi, les premiers, ont fait état en 1985 du faible taux de participation des Franco-Ontariens à l'enseignement supérieur, le situant à la moitié de celui des nonfrancophones de la province ${ }^{22}$. Dans une analyse subséquente pour la période de 1979 à 1989, Frenette et Quazi ont relevé deux moments clés de déséquilibre qui contribuaient à ce faible taux de participation : d'abord, lors de la transition entre la $12^{\mathrm{e}}$ et la $13^{\mathrm{e}}$ année où le taux des francophones à accéder à la $13^{\circ}$ année était moins élevé, puis lors de la transition entre l'obtention du diplôme d'études secondaires et l'entrée à l'université, les francophones étant, ici aussi, moins nombreux à s'inscrire à l'universitée 23 .

Dans une enquête plus récente parue en 2009, Normand Labrie, Sylvie Lamoureux et Denise Wilson ${ }^{24}$ révèlent que le nombre de finissants francophones du secondaire à demander l'entrée à l'université a légèrement augmenté de 1998 à 2006. Leur calcul ne tient cependant compte que des admissions directes des diplômés du secondaire, ce qui ne comprend pas les individus qui ne demandent pas l'admission immédiatement à la fin de leurs études secondaires. De même, Jean-Pierre Corbeil et Sylvie Lafrenière ${ }^{25}$ soulignent la diminution des écarts

22. Stacy Churchill, Saeed Quazi et Normand Frenette, Le Diagnostic d'un système d'éducation. Vol. 2 : L'enseignement postsecondaire. Rapport statistique, Toronto, Conseil de l'éducation franco-ontarienne, 1985.

23. Normand Frenette et Saeed Quazi, « La francophonie ontarienne et l'accès à l'enseignement supérieur», Francophonies d'Amérique, no 4, 1994, p. 15, www. erudit.org (consulté le 17 novembre 2014).

24. Normand Labrie, Sylvie Lamoureux et Denise Wilson, L'Accès des francophones aux études postsecondaires en Ontario : le choix des jeunes, OISE / Centre de recherches en éducation franco-ontarienne, University of Toronto, 2009, s.p. Voir aussi Ontario, "L'accès des francophones aux études postsecondaires en Ontario : le choix des jeunes ", De la théorie à la pratique, Monographie $n^{\circ} 13$, Direction des politiques et programmes de langue française, Ministère de l'Éducation de l'Ontario et CFORP, novembre 2011.

25. Jean-Pierre Corbeil et Sylvie Lafrenière, Portrait des minorités de langue 
de fréquentation du postsecondaire par les francophones et les anglophones de l'Ontario et parlent d'un rattrapage considérable depuis 2006 chez les 25 à 34 ans. Mary Catharine Lennon et ses collaborateurs corroborent ce fait en soulignant qu'une proportion équivalente d'Anglo-Ontariens et de Franco-Ontariens ne font pas d'études postsecondaires (13\% chez les premiers et $14 \% \mathrm{chez}$ les seconds $)^{26}$. Cependant, ces études ne tiennent pas compte de la langue dans laquelle a lieu la scolarité. Ainsi, bien qu'elles indiquent que de plus en plus de jeunes francophones fréquentent les établissements postsecondaires et que l'écart qui sévit depuis des décennies s'atténue, voire s'estompe, il est toujours difficile de savoir dans quelle langue ils étudient. Il peut donc s'agir d'une amélioration du niveau d'instruction, mais il se peut également que celle-ci se fasse au détriment de la langue maternelle.

L'enquête menée par Labrie et ses collaborateurs laisse entrevoir le type d'établissement - collégial ou universitaire - vers lequel s'orientent les jeunes ressortissants des écoles francoontariennes et la langue dans laquelle ils choisissent de faire leurs études postsecondaires. Au cours de la période de 1998 à 2006, 40887 jeunes francophones ont fait des études postsecondaires ; sur ce nombre, 22729 (55,6 \%) ont choisi d'étudier en français, tandis que $18158(44,4 \%)$ ont opté pour l'anglais. Selon les auteurs, ce passage à l'anglais se serait opéré au secondaire pour $2779(6,8 \%)$ d'entre eux ${ }^{27}$. Sur l'ensemble des francophones à

officielle au Canada: les francophones de l'Ontario, Ottawa, Ministre de l'Industrie, Statistique Canada, 2010, n' $89-642-X$ au catalogue.

26. Mary Catharine Lennon, Huizi Zhao, Shunji Wang et Tomasz Gluszynki, Facteurs influençant l'accès des jeunes à l'éducation postsecondaire en Ontario, Toronto, Conseil ontarien de la qualité de l'enseignement supérieur, 2011, 25 p.

27. Suite à une étude sur le décrochage scolaire chez les francophones de l'Ontario, Gratien Allaire et ses collaborateurs ont décelé le passage des élèves des écoles franco-ontariennes vers les écoles de langue anglaise : Gratien Allaire, Jacques Michaud, Julie Boissonneault, Daniel Côté et Pounthioun Diallo, Le Décrochage au secondaire en Ontario français : le point de vue des jeunes, Rapport soumis par l'Institut franco-ontarien, Université Laurentienne, à la Direction des politiques et programmes d'éducation en langue française, Ministère de l'Éducation de l'Ontario, octobre 2005, $63 \mathrm{p}$. Ces transferts semblaient s'opérer surtout lors des transitions de la $6^{\mathrm{e}}$ à la $7^{\mathrm{e}}$, et de la $8^{\mathrm{e}}$ à la $9^{\mathrm{e}}$ année. Julie Boissonneault et Simon Laflamme ont mené en 2015 une enquête provinciale sur ce qui motive ce passage dans la langue de la scolarité 
avoir fait des études postsecondaires, près des deux tiers $(64,4 \%)$ se sont orientés vers le collégial et un peu plus du tiers $(35,6 \%)$ vers l'universitaire, mais les données ne permettent pas de savoir combien d'entre eux ont choisi, en fait, d'étudier en français à l'université.

Stéphanie Garneau et Janelle Comtois (2009) se sont intéressées, elles aussi, à l'accès aux études postsecondaires des étudiants francophones de l'Ontario dans le contexte de la transformation qui s'opère au sein des universités dans les sociétés occidentales où technicisation et gestion managériale prennent le dessus sur l'université qui est de moins en moins un lieu de réflexion critique, de débats sociaux et d'universalité de la scientificité. L'université, l'ultime aboutissement de l'enjeu dans la production et le maintien culturo-linguistique, est, à leurs yeux, « aujourd'hui traversée, dans ce milieu minoritaire, d'une tension contradictoire entre une mission culturelle qui se traduit par la contribution au développement de la francophonie et une mission économique qui consiste à répondre aux besoins du marché ${ }^{28}$ "».

Il importe donc de mieux comprendre ce qui incite les jeunes francophones à faire des études postsecondaires et, de surcroît, à les faire en français ou en anglais. C'est l'objet de notre réflexion.

\section{II - ChoiX de la LANGue D'ENSEIGNement À L'UNIVERSITÉ}

Que savons-nous de ce qui encourage ou de ce qui entrave la fréquentation des établissements postsecondaires par les FrancoCanadiens en situation minoritaire et par les francophones de l'Ontario ? De ce qui les motive, lorsqu'ils décident de fréquenter le collège ou l'université, à choisir d'étudier en français ou en anglais ?

aux paliers élémentaire et secondaire. Voir Julie Boissonneault et Simon Laflamme, Passage des élèves de l'école franco-ontarienne vers l'école de langue anglaise, Rapport de recherche présenté au Centre canadien de leadership en évaluation et au ministère de l'Éducation de l'Ontario, $1^{\text {er }}$ mars 2016, 78 p.

28. Stéphanie Garneau et Janelle Comtois, «Les jeunes Franco-Ontariens et l'accès à l'enseignement supérieur à l'heure de l'université entrepreneuriale », Reflets, Revue d'intervention sociale et communautaire, vol. 15, 2009, p. 2-3. 
Dans l'analyse qu'ils font des demandes d'admission aux établissements postsecondaires pour la période de 1998 à 2006, Labrie, Lamoureux et Wilson constatent que le facteur le plus déterminant est celui de la proximité des établissements. Ils corroborent ainsi les recherches précédentes ${ }^{29}$, qui laissent entrevoir « un préjugé favorable » à l'égard d'établissements situés près du foyer. Près des deux tiers (65\%) des étudiants qui s'orientent vers le collège et plus de la moitié ( $53 \%$ ) de ceux qui visent l'université se sont inscrits, de 1998 à 2006, à un établissement situé à moins de 75 kilomètres de leur domicile. Selon ces chercheurs, la proximité est plus déterminante que ne l'est le programme d'études et la langue de la scolarité lorsque les jeunes s'orientent vers le collégial, mais elle le serait moins lorsqu'il s'agit d'études universitaires. Dans les deux universités les plus fréquentées par les finissants des écoles secondaires franco-ontariennes que sont l'Université d'Ottawa et 1'Université Laurentienne ${ }^{30}$, le taux de fréquentation par des étudiants habitant à moins de 75 kilomètres de l'établissement est de $58 \%$ pour celle-là et de $59 \%$ pour celle-ci ${ }^{31}$.

Dans une enquête menée en 2012-2013 auprès des élèves de $12^{\mathrm{e}}$ année dans le but de cerner les facteurs qui influent sur le choix de programme postsecondaire ou vocationnel et sur la langue dans laquelle ils ont l'intention d'étudier, André Samson ${ }^{32}$ met en relief l'importance de la décision que l'élève aura à prendre. À son avis, trois grands facteurs interviennent sur la justesse et la pertinence du choix, à savoir la connaissance que l'élève a de lui-même (ses intérêts, ses aptitudes, ses valeurs), la connaissance qu'il a du marché (par les conseils qu'il reçoit du milieu scolaire

29. Normand Frenette, Accès au collège et à l'université : est-ce que la distance importe? Ottawa, Statistique Canada, 2003.

30. L'Université d'Ottawa attirerait $55 \%$ des étudiants francophones et l'Université Laurentienne, $15 \%$, selon Normand Labrie et collaborateurs, op. cit.

31. Normand Labrie et collaborateurs, op. cit.

32. André Samson, Les Transitions scolaires des études secondaires vers les programmes de formation postsecondaire: une étude qualitative et quantitative, Rapport de recherche, Université d'Ottawa, 2014, p. 13, www.education.uottawa.ca (consulté le 20 février 2015). 
et les recherches qu'il fait) et la capacité qu'il a à prendre une décision. Pour ce qui est de la langue de la scolarité, l'étude de Samson soutient :

que le choix de poursuivre des études postsecondaires en français est un prolongement de la construction identitaire et vocationnelle de l'élève. En effet, et contrairement à Labrie et collaborateurs, ce n'est pas uniquement le facteur géographique qui détermine le choix d'un programme d'études postsecondaires en français. Ce choix est aussi et surtout déterminé par le sentiment de compétence en français, le sentiment d'appartenance à la francophonie et une connaissance de la qualité des institutions d'enseignement francophones ou bilingues ${ }^{33}$.

Samson relève néanmoins des différences régionales quant à l'intention des jeunes de faire des études postsecondaires en français : 76,2\% des jeunes de l'Est ontarien étudient en français, contre $32,7 \%$ des jeunes du Sud et 37,2 \% de ceux du Nord qui font de même. Le choix est corrélé à plusieurs autres variables, dont la connaissance qu'ils ont des possibilités qui s'offrent à eux (cela étant déterminé par des discussions avec des conseillers d'orientation et leurs recherches personnelles sur la toile) et le soutien qu'ils reçoivent du milieu familial et scolaire. « Plus les répondants optent de poursuivre leurs études postsecondaires en français, plus leur sentiment de bien-être subjectif est élevée ${ }^{34} »$.

Pour Samson, la proximité d'un établissement bilingue ou de langue française ne vient qu'au cinquième rang $(11,8 \%)$ à l'échelle provinciale lorsqu'il analyse les variables les plus susceptibles d'agir sur le choix de faire des études postsecondaires en français. Le premier facteur serait l'aisance à étudier en français $(14,5 \%)$, le deuxième, la qualité des programmes d'études dans les établissements (14\%), le troisième, la possibilité de se trouver un bon emploi qui exige le bilinguisme $(13,5 \%)$ et, le quatrième, la fierté d'être francophone (13,3\%). En contrepartie, les facteurs qui agissent sur la motivation à étudier en anglais sont, en ordre décroissant à l'échelle provinciale, la reconnaissance et le

33. Ibid., p. v.

34. Ibid., p. 25. 
prestige des établissements (20,1\%), le manque de programmes d'études en français (19\%), la possibilité de se trouver un bon emploi (18,3\%), le manque de compétence en français $(15,7 \%)$ et l'éloignement des établissements bilingues ou francophones $(11,5 \%)$. Il précise néanmoins que « les répondants qui décident de poursuivre leurs études postsecondaires en anglais le font surtout parce que leur programme n'est pas proposé ou parce qu'ils perçoivent que les institutions d'enseignement anglophones sont plus prestigieuses ${ }^{35} »$.

Ces résultats rejoignent ceux de l'enquête menée, au début des années 2000, auprès de jeunes étudiants albertains, par Maud Lemaire et Cameron Montgomery ${ }^{36}$, lesquels relevaient trois facteurs d'intervention sur le choix de la langue d'enseignement à l'université : l'intensité du vécu en français dans leur environnement (en famille, à l'école et dans le milieu socio-institutionnel), le rapport entre leur socialisation et leur appartenance identitaire, ainsi que le lien qu'ils tissaient entre langue, culture et identité. Selon Lemaire et Montgomery, ceux qui choisissent d'étudier en anglais le font pour des raisons utilitaires (rester avec leurs amis, compétence dans les deux langues, qualité de l'enseignement, plus grandes possibilités de services et meilleurs débouchés sur le marché du travail), alors que ceux qui optent pour l'université en langue française justifient davantage leur choix par des facteurs culturels (améliorer leur français qu'ils craignent de perdre et se réapproprier leur identité culturelle à travers la langue).

[D]es compétences insuffisantes ou perçues par l'individu comme telle [sic] en français, la distance de l'université à fréquenter, le nombre de programmes et de services restreints par rapport aux universités anglophones sont des facteurs inhérents à la structure de l'université de la minorité pouvant faire préférer le choix de l'anglais comme langue d'enseignement à ce niveau de la scolarité ${ }^{37}$.

35. Ibid., p. v.

36. Maud Lemaire et Cameron Montgomery, op. cit.

37. Ibid. 
Ces recherches mettent ainsi de l'avant le fait que les motivations à choisir d'étudier en français jouent sur plusieurs tableaux en même temps. Les facteurs de la proximité géographique des établissements postsecondaires où il y a des programmes en français et le sentiment de compétence langagière en français y sont présentés comme des vecteurs importants qui agissent sur la décision que prennent les francophones minoritaires de la province d'étudier en français. Ces deux facteurs relèvent à la fois de l'utilitaire et du culturel, de l'institutionnel et de l'individuel. La question est complexe.

Pour mieux comprendre la situation, nous avons analysé le discours tenu par un groupe d'étudiants inscrits à l'Université Laurentienne en 2005, afin de voir s'ils faisaient appel à ces facteurs pour expliquer le choix de leur langue d'instruction. À l'instar de ces chercheurs, ce qui nous intéresse, c'est de mieux comprendre les raisons pour lesquelles les jeunes francophones de l'Ontario étudient ou non dans leur langue maternelle.

\section{III - QUE DISENT LES ÉTUDIANTS ?}

Les données recueillies permettront d'examiner le choix de la langue dans laquelle ils ont décidé de faire leurs études et de mieux comprendre ce choix. L'enquête, dont le but premier était de cerner les divergences et les convergences dans les représentations $\mathrm{du}$ bilinguisme et des deux langues officielles selon la langue maternelle ${ }^{38}$, vérifiait, entre autres, la langue dans laquelle ils avaient été scolarisés (de la $1^{\text {re }}$ année à la fin du secondaire), le choix de la langue de leurs études postsecondaires et la raison de ce choix, en tenant compte de variables sociodémographiques et sociolinguistiques.

Bien que ces données soient propres à un environnement institutionnel spécifique et qu'elles s'inscrivent dans un moment précis, elles peuvent lever le voile sur ce qui motive le choix de

38. Julie Boissonneault, « Divergences et convergences dans les représentations du bilinguisme ", Francophonies d'Amérique, n 25, 2008, p. 19-48, www. erudit.org. 
la langue d'instruction au postsecondaire puisque la voix des premiers intéressés à ce sujet - les étudiants -, quoique succincte, y est recueillie par le biais d'une question ouverte.

Sur les 326 questionnaires distribués en salle de classe auprès d'un échantillon non probabiliste de répondants parlant l'anglais, le français ou les deux langues, nous n'en retenons que 210 : ceux qui ont déclaré le français comme leur ou l'une de leurs langues maternelles $\left(\mathrm{L}_{1}\right)$ et ceux qui ont fait en tout ou en partie leur scolarité à l'élémentaire et au secondaire en français ${ }^{39}$. Ces variables sont, à notre avis, celles qui agissent le plus sur la possibilité d'étudier dans une langue plutôt que dans une autre. Il est ainsi plus probable qu'un étudiant se scolarise dans sa langue maternelle que dans une autre langue, tout comme il est plus probable qu'un étudiant scolarisé dans une langue donnée continue ses études dans cette langue. C'est donc dire qu'il est peu probable qu'un individu fasse des études dans une langue qui n'est pas sa première langue de communication et dans laquelle il n'a jamais été scolarisé.

\section{Profil des répondants}

Dans l'ensemble des 210 répondants, 144 (68,6 \%) étudiants déclarent avoir le français comme seule langue maternelle. À ce nombre, s'ajoutent 4 (1,9\%) étudiants pour qui le français est l'une des langues maternelles, 60 (28,6 \%) étudiants qui sont locuteurs natifs ${ }^{40}$ de l'anglais et 2 étudiants $(1,0 \%)$ qui ont une langue maternelle autre que le français ou l'anglais. Ces étudiants ont tous en commun le fait d'avoir fait l'entièreté ou une partie de leurs études élémentaires et secondaires dans des écoles de langue française ou dans des écoles d'immersion française.

Leur parcours scolaire, toutefois, ne s'inscrit pas dans un tracé immuable, comme l'illustre le tableau 1. Des facteurs propres à la

39. Sont ainsi retirés de l'étude les 116 répondants qui n'ont pas le français comme langue maternelle (ou comme l'une de leur langue maternelle) et qui n'ont fait aucune scolarité en français (autre que les cours de français $\mathrm{L}_{2}$ obligatoires dans le curriculum provincial).

40. Nous entendons par « locuteur natif», l'individu qui a acquis une langue en tant que langue maternelle et qui la parle couramment. 
région (présence et type d'écoles), à la famille (déménagement) et à l'individu (conflits et difficultés scolaires) interviennent et modifient le parcours des élèves de sorte que la langue d'instruction n'est pas toujours la même au fil des ans.

\section{Tableau 1}

Distribution des répondants selon les années de scolarité de l'élémentaire et du secondaire et selon la langue principale d'instruction

\begin{tabular}{|l|c|c|c|c|c|c|}
\hline & $\begin{array}{c}\text { Uniquement } \\
\text { en français } \\
\mathrm{N}(\%)\end{array}$ & $\begin{array}{c}\text { Plutôt en } \\
\text { français }^{41} \\
\mathrm{~N}(\%)\end{array}$ & $\begin{array}{c}\text { Autant } \\
\text { qu'en anglais } \\
\mathrm{N}(\%)\end{array}$ & $\begin{array}{c}\text { Plutôt en } \\
\text { anglais }^{42} \\
\mathrm{~N}(\%)\end{array}$ & $\begin{array}{c}\text { Uniquement } \\
\text { en anglais } \\
\mathrm{N}(\%)\end{array}$ & Total \\
\hline $1^{\text {re }}$ année & $180(85,7)$ & $19(9,0)$ & $6(2,9)$ & $2(1,0)$ & $3(1,4)$ & 210 \\
\hline $2^{\mathrm{e}}$ année & $177(84,3)$ & $24(11,4)$ & $5(2,4)$ & $2(1,0)$ & $2(1,0)$ & 210 \\
\hline $3^{\mathrm{e}}$ année & $162(77,1)$ & $40(19,0)$ & $5(2,4)$ & $2(1,0)$ & $1(0,5)$ & 210 \\
\hline $4^{\mathrm{e}}$ année & $150(71,4)$ & $46(21,9)$ & $11(5,2)$ & $3(1,4)$ & & 210 \\
\hline $5^{\mathrm{e}}$ année & $140(66,7)$ & $53(25,2)$ & $15(7,1)$ & $2(1,0)$ & & 210 \\
\hline $6^{\mathrm{e}}$ année & $138(65,7)$ & $53(25,2)$ & $17(8,1)$ & $2(1,0)$ & & 210 \\
\hline $7^{\mathrm{e}}$ année & $132(62,9)$ & $49(23,3)$ & $27(12,9)$ & $1(0,5)$ & & 209 \\
\hline $8^{\mathrm{e}}$ année & $133(63,3)$ & $48(22,9)$ & $27(12,9)$ & $1(0,5)$ & & 209 \\
\hline $9^{\mathrm{e}}$ année & $130(61,9)$ & $43(20,5)$ & $27(12,9)$ & $7(3,3)$ & $3(1,4)$ & 210 \\
\hline $10^{\mathrm{e}}$ année & $129(61,4)$ & $41(19,5)$ & $27(12,9)$ & $10(4,8)$ & $3(1,4)$ & 210 \\
\hline $11^{\mathrm{e}}$ année & $127(60,5)$ & $39(18,6)$ & $27(12,9)$ & $13(6,2)$ & $4(1,9)$ & 210 \\
\hline $12^{\mathrm{e}}$ année & $125(59,5)$ & $38(18,1)$ & $27(12,9)$ & $14(6,7)$ & $4(1,9)$ & 208 \\
\hline CPO & $54(25,7)$ & $16(7,6)$ & $19(9,0)$ & $9(4,3)$ & $5(2,4)$ & $107^{43}$ \\
\hline
\end{tabular}

Ainsi, sur les 180 répondants qui commencent l'école uniquement en français, 135 (75\%) ont le français comme seule langue maternelle et $42(23 \%)$ sont locuteurs natifs de l'anglais. Que se produit-il pour que, en $12^{\mathrm{e}}$ année, ils ne soient que 125 (dont 106 locuteurs natifs du français - 59\% de la cohorte initiale - et

41. Il peut y avoir une ambiguïté entre dire étudier « uniquement » ou «plutôt » dans une langue. La nuance se comprend s'il s'agit d'école d'immersion ou s'il y a changement d'école, mais non si l'élève demeure dans une école de langue française. La formulation de la question précisait de ne pas tenir compte des cours de langue, mais il se peut que cela n'ait pas été compris.

42. Voir note précédente.

43. Le nombre peu élevé d'étudiants à avoir fait le CPO (cours préuniversitaire de l'Ontario, auparavant connu comme la $13^{\mathrm{e}}$ année) s'explique du fait qu'il a été supprimé du système scolaire ontarien en 2002. Plusieurs des étudiants de l'échantillon ont ainsi été admis à l'université à la fin de leur $12^{\mathrm{e}}$ année. 
17 locuteurs natifs de l'anglais) à terminer leur secondaire uniquement en français? Il est impossible de répondre à cette question.

Chez les uns et chez les autres, la diminution s'explique par l'accroissement du nombre de répondants qui déclarent étudier « plutôt en français » ou « autant en français qu'en anglais » au fil des ans. Il ne peut s'agir, à notre avis, que du transfert vers des écoles d'immersion française, quoique le questionnaire ne permette pas de le vérifier. Chez les répondants anglophones, il semble se produire aussi, surtout à partir de la $9^{\mathrm{e}}$ année, un transfert vers l'anglais comme langue principale d'enseignement, ici aussi soit vers des écoles d'immersion, soit vers des écoles de langue anglaise. Ce type de passage a été relevé dans des études antérieures $^{44}$ et a fait, en 2015, l'objet d'une enquête provinciale ${ }^{45}$.

Que pouvons-nous dégager de ce tableau ? Que ces 210 répondants, tous inscrits à l'Université Laurentienne en 2005, auraient pu choisir de faire en tout ou en partie leurs études postsecondaires en français puisque le français est leur langue maternelle ou l'une de leurs langues maternelles et qu'ils ont étudié, à un moment ou à un autre, en français dans une école de langue française ou dans une école d'immersion française.

\section{Choix de la langue de la scolarité à l'université}

Nous nous intéressons, rappelons-le, à ce qui motive le choix de la langue de la scolarité chez les francophones à l'université. Nous avancions que seraient plus enclins à étudier en français les étudiants de langue maternelle française ou les étudiants ayant fait leur scolarité antérieure entièrement ou partiellement en français. Or, sur les 210 répondants qui correspondaient à ces critères, moins de la moitié $(41,4 \%)$ déclarent faire des études universitaires « uniquement en français », le quart (25,7\%) estime les faire « plutôt en français », 8,6 \% disent étudier « autant en

44. Voir Gratien Allaire et collaborateurs, op. cit. ; Simon Laflamme, « Passage de l'élémentaire au secondaire et décrochage culturel en Ontario français. Élimination de quelques facteurs ", Cahiers Charlevoix 9. Études franco-ontariennes, Ottawa, Presses de l'Université d'Ottawa, 2012, p. 15-59.

45. Julie Boissonneault et Simon Laflamme, op. cit. 
français qu'en anglais », alors que près du quart $(24,3 \%)$ étudient soit « plutôt en anglais » $(9,5 \%)$, soit « uniquement en anglais » $(14,8 \%)$. Le tableau 2 illustre combien de ces étudiants font leurs études universitaires dans leur langue première et combien les font dans leur langue seconde.

\section{Tableau 2}

Langue d'instruction à l'université selon la langue maternelle ${ }^{46}$

\begin{tabular}{|l|rr|rr|}
\hline & \multicolumn{4}{|c|}{ Langue maternelle $\left(\mathrm{L}_{1}\right)$} \\
\hline Langue d'instruction à l'université & \multicolumn{2}{|c|}{$\begin{array}{l}\text { Français } \\
\text { seule } \mathrm{L}_{1} \\
\mathrm{~N}(\%)\end{array}$} & $\begin{array}{c}\text { Anglais } \\
\text { seule } \mathrm{L}_{1} \\
\mathrm{~N}(\%)\end{array}$ \\
\hline Uniquement en français & 75 & $(52,1)$ & 10 & $(16,7)$ \\
\hline Plutôt en français & 43 & $(29,9)$ & 8 & $(13,3)$ \\
\hline Autant en français qu'en anglais & 11 & $(7,6)$ & 6 & $(10,0)$ \\
\hline Plutôt en anglais & 8 & $(5,6)$ & 12 & $(20,0)$ \\
\hline Uniquement en anglais & 7 & $(4,9)$ & 24 & $(40,0)$ \\
\hline & 144 & $(100,0)$ & 60 & $(100,0)$ \\
\hline
\end{tabular}

La moitié des répondants qui ont le français comme langue maternelle unique $(52,1 \%)$ font leurs études universitaires « uniquement en français » et plus du quart $(29,9 \%)$ les font « plutôt en français ». C'est donc dire que $82 \%$ des francophones de l'échantillon étudient essentiellement en français. Les explications qu'ils donneront quant au choix de la langue de la scolarité feront la lumière sur la distinction qu'ils établissent entre étudier « uniquement » ou «plutôt» en français. Nous y reviendrons. Dix-huit pour cent (18\%) d'entre eux, toutefois, ont choisi, dans une plus ou moins grande mesure, d'étudier en anglais. Nous verrons ultérieurement s'il s'agit d'un choix voulu ou d'un choix imposé par des circonstances externes.

Sur les 144 étudiants ayant le français comme seule langue maternelle, 142 (98,7\%) ont commencé l'école élémentaire ( $1^{\text {re }}$ année) uniquement ou plutôt en français et, à la fin du secon-

46. Nous ne présentons pas les six répondants qui ont déclaré avoir plus d'une langue maternelle puisqu'ils ne sont pas suffisamment nombreux. 
daire $\left(12^{\mathrm{e}}\right.$ année $\left.{ }^{47}\right), 135$ (95\%) étudiaient toujours uniquement ou plutôt en français. Dans cette cohorte, 112 (78 \%) ont choisi de faire leurs études universitaires en français.

Chez les 60 étudiants pour qui l'anglais est la seule langue maternelle, il y a plus de va-et-vient quant à la langue de scolarité préalable. Bien que la majorité d'entre eux (85\%) commencent l'école élémentaire en français, seulement 24 (40\%) étudient encore « uniquement» ou « plutôt» en français à la fin du secondaire. Les autres se distribuent équitablement entre des études « autant en français qu'en anglais » $(30 \%, \mathrm{n}=18)$ et des études « plutôt » ou « uniquement » en anglais (30\%, $\mathrm{n}=18)$.

Il est intéressant de noter que $30 \%$ des anglophones de notre échantillon font leurs études postsecondaires essentiellement en français. Du fait que ces individus aient été scolarisés antérieurement en partie ou entièrement en français, ils ne peuvent être généralisés à l'ensemble des locuteurs natifs de l'anglais, qu'ils soient monolingues ou bilingues. Le fait qu'une plus grande proportion d'anglophones (30\%) que de francophones (10,4\%) de cet échantillon étudient dans leur langue seconde ne doit également pas être présenté comme le reflet d'une plus grande ouverture de leur part à l'égard de leur langue seconde, ni d'une meilleure connaissance ou d'une plus grande maîtrise de cette dernière. Ce serait là un jugement hâtif et probablement erroné, puisqu'il y a, nous le rappelons, des enjeux sociaux différents entre étudier dans une langue majoritaire détentrice d'un statut officiel et le faire dans une langue minoritaire sans statut officiel, selon que l'on soit locuteur natif de celle-ci ou de celle-là. C'est donc dire qu'étudier dans sa langue seconde ne revêt pas la même signification lorsque cette dernière est la langue de la majorité ou celle de la minorité. Dans cet échiquier, les langues n'ont pas le même poids ${ }^{48}$ - c'est-à-dire la même importance et la même

47. Tous les répondants ont dû faire la $12^{\mathrm{e}}$ année, mais seulement ceux de $3^{\mathrm{e}}$ ou $4^{\mathrm{e}}$ année de leur programme universitaire ont fait le cours préuniversitaire de l'Ontario (CPO). Nous avons donc choisi de ne pas en tenir compte.

48. Nous entendons « poids » ici en termes de représentations et non en termes mesurables. Cette représentation s'inscrit dans un processus sociopolitique. Le 
valeur dans l'imaginaire collectif - et ce déséquilibre se traduit dans les usages qu'on en fait. La perception de ce déséquilibre pourrait être l'un des facteurs qui agit sur la langue de la scolarité au postsecondaire par les locuteurs de la langue minoritaire.

\section{Justification du choix de la langue d'instruction}

Une question ouverte invitait les répondants à expliquer ou à justifier le choix de la langue d'instruction universitaire, ce qui permet de mieux comprendre leurs motivations à étudier ou non en français. S'agira-t-il, comme nous l'avions soulevé antérieurement, de motivations d'ordre utilitaire et pragmatique (définies, entre autres, par des facteurs socioéconomiques ou d'accessibilité) ou de motivations de nature culturelle et idéologique ? Leurs discours seront-ils empreints d'un jugement métalinguistique (facilité ou difficulté inhérente attribuée à la langue - le français ou l'anglais) ? D'un jugement sur la communauté linguistique (utilité et vitalité sociales) ou d'un sentiment d'appartenance à cette communauté ? C'est ce que nous cherchons à dégager.

Il se peut également que la langue (ou les langues) dans laquelle ils étudient ne corresponde pas à la langue (ou aux langues) dans laquelle ils auraient voulu le faire. C'est aussi ce que nous avons cherché à cerner. S'agit-il toujours, chez les francophones, d'une volonté manifeste, d'un choix voulu, de continuer leurs études dans leur langue maternelle ? Si tel n'est pas le cas, et qu'il s'agit d'un choix imposé par les circonstances, quels facteurs viennent perturber la volonté et le désir initial des répondants? L'analyse tentera de répondre à ces questions.

\section{a) Francophones qui étudient uniquement en français}

Trois grandes motivations se dégagent du discours des répondants francophones qui ont dit étudier « uniquement en français ». Sur le plan utilitaire, ils font valoir leur compétence langagière en

concept métaphorique du «poids des langues » est une construction polysémique et multidimensionnelle. Pour en savoir davantage, voir Médéric Gasquet-Cyrus et Cécile Petitjean (dir.), Le Poids des langues. Dynamiques, représentations, contacts, conflits, Paris, L'Harmattan, coll. « Espaces discursifs », 2009, 320 p. 
français, elle-même attribuable au fait qu'il s'agit de leur langue maternelle, mais aussi de la langue dans laquelle ils ont antérieurement été scolarisés, d'une part, et leurs visées professionnelles, d'autre part. Sur le plan culturel, ils expliquent leur choix par des critères socioaffectifs liés à la langue. Ces motivations, comme l'illustrent les énoncés reproduits, ne sont pas étanches l'une à l'autre.

\section{Compétence langagière}

La motivation la plus récurrente est celle de la compétence en langue française, tout au plus, d'une plus grande aisance à s'exprimer en français qu'en anglais, bien que la grande majorité des répondants francophones s'estiment bilingues $(98,6 \%)$, ayant expressément déclaré parler anglais. Le tiers $(n=24)$ des 75 répondants parle surtout d' " être à l'aise » ou d'être plus « confortable » en français, ce qui pourrait dénoter beaucoup plus qu'une compétence strictement linguistique, mais également discursive qui tienne compte du comportement langagier.

Je me sens plus à l'aise et confortable en français qu'en anglais. (énoncé 137, ID 75)

J'ai choisi de ne pas prendre des cours en anglais, car je ne me sens pas assez à l'aise pour prendre un ou des cours universitaires anglophones. Je ne le pratique pas assez. J'ai commencé un cours en "Philosophy» et je ne pouvais pas continuer, car je me sentais perdue. (énoncé 4, ID 80)

Je comprends mieux lors des sessions de classes en français. (énoncé 68, ID 8)

Je suis plus à l'aise en français pour communiquer et avoir fait mes cours en anglais aurait fait chuter mes notes. (énoncé 26, ID 97)

Le fait que la scolarité précédant l'entrée à l'université ait été en langue française contribue aussi, à leurs yeux, à continuer dans cette langue. D'aucuns précisent qu'il s'agit d'une suite logique, d'autant qu'ils ont pris l'habitude de rédiger leurs travaux dans cette langue. 
Je suis plus confortable, car j'ai toujours étudi [é] en français. Les mots et les termes me vienne[nt] plus facilement. (énoncé 7, ID 82)

Je préfère le français. J'ai toujours pris des cours en français et je n'ai vu aucune raison de changer. (énoncé 69, ID 9)

$J$ 'ai toujours fait mes études scolaires en français, alors j'ai continué pour mes années collégiales et universitaires (à part d'un seul cours anglais obligatoire). (énoncé 87, ID 29)

\section{Motivations socioprofessionnelles ou socioéconomiques}

Quatorze énoncés font état de motivations socioprofessionnelles. C'est d'abord et avant tout le cas des étudiants qui se destinent à l'enseignement dans les écoles de langue française, et ils sont nombreux. Puisque la formation universitaire pour obtenir le baccalauréat en éducation et le brevet d'enseignement qui permet d'enseigner dans les écoles franco-ontariennes doit se faire en français et puisque le français, comme discipline, y est aussi une matière scolaire, les étudiants qui envisagent une carrière en enseignement dans les écoles de langue française doivent forcément se former dans cette langue. Ces étudiants de premier cycle doivent ainsi suivre tous leurs cours obligatoires en français.

Je planifie d'enseigner en français, donc je veux apprendre ma matière d'enseignement en français. (énoncé 95, ID 36)

Je veux enseigner [plus] tard en français et mon cours oblige que tous mes cours soient en français. (énoncé 45, ID 117)

Je souhaite devenir une enseignante de français au secondaire. De plus, je me sens beaucoup plus à l'aise en français. (énoncé 41, ID 110)

Étudier en français comporte aussi, à leurs yeux, certains avantages socioéconomiques, la connaissance des deux langues officielles du pays étant un atout sur le marché du travail.

Je crois que c'est un avantage d'étudier en français, car les employeurs recherche[nt] les gens bilingues. (énoncé 5, ID 81)

Les analyses précédentes de cette enquête avaient d'ailleurs fait valoir l'importance que les francophones accordent à la repré- 
sentation du bilinguisme comme un avantage sur le marché du travail. Bien que les anglophones reconnaissent aussi le bilinguisme comme un atout important pour le marché du travail, les représentations des uns et des autres variaient en ce que les francophones le voyaient davantage comme une compétence incontournable, tandis que les anglophones le percevaient comme une valeur ajoutée ${ }^{49}$.

\section{Motivations socioaffectives}

Les répondants francophones qui étudient "uniquement » en français véhiculent aussi dans leur discours des propos d'ordre socioaffectif liés à la langue. Nous avons relevé 18 énoncés qui se rapportent à l'expression du sens d'appartenance à une communauté linguistique et au sens d'identité (franco-ontarienne et canadienne-française), qui ont un rapport à la culture exprimée par la langue maternelle ou qui expriment une prise de position ou de conscience politique sur les enjeux sociolinguistiques associés au fait de vivre en situation minoritaire.

Je suis fière d'être franco-ontarienne et je désire garder ma langue et ma culture. (énoncé 64, ID 5)

Je crois que [c'est] important de continuer la culture française et la langue est une grosse partie de ceci. (énoncé 33, ID 104) Je suis française, je comprends très bien le français, c'est ma langue maternelle et je suis fière. (énoncé 136, ID 74)

Je préfère garder ma langue maternelle comme langue première. Je veux devenir enseignante en français. (énoncé 301, ID 113)

J'étudie en français parce que pour moi je me sens plus confortable, mais aussi il faut supporter notre langue sinon tous ces cours vont disparaître et même [l']université en français. (énoncé 84, ID 24)

\section{b) Francophones qui étudient plutôt en français}

Plus du quart des étudiants de langue maternelle française de

49. Julie Boissonneault, op. cit. 
notre échantillon $(29,9$ \%) déclare étudier « plutôt en français ». Tiennent-ils le même discours que ceux qui étudient « uniquement en français »? À quelques exceptions près, non. Leur discours permet de comprendre que la nuance qu'ils établissent entre étudier « uniquement » en français et le faire « plutôt» en français découle du fait qu'ils suivent des cours dans les deux langues.

J'ai trois cours en français et deux cours en anglais. (énoncé 10, ID 85)

Trois de mes 5 cours sont en français. (énoncé 81, ID 21)

J'ai présentement 5 cours de 6 crédits. Deux de ces cours se passent en anglais (English et COSC 1701-ordinateur) et les trois autres se passent en français (grammaire, sociologie et psychologie). (énoncé 138, ID 76)

Dans la majorité des cas, ils précisent ne pas étudier uniquement en français en raison du manque de cours en langue française. Et ils sont nombreux à le dire. Vingt-cinq de leurs énoncés - plus de la moitié des justifications avancées - sont explicites à cet égard.

Il n'y a pas grand choix de cours de Science politique offert [s] en français donc je prends un cours de $3^{e}$ année en anglais. (énoncé 21, ID 92)

J'aurais voulu, mais un de mes cours obligatoires s'offrait exclusivement en anglais. (énoncé 37, ID 107)

Certains cours nécessaires à l'obtention de mon baccalauréat ne sont offert[s] qu'en anglais. (énoncé 77, ID 18)

Je prends tou[s] mes cours en français, tandis que j'ai dî prendre des cours d'ordinateur en anglais. (énoncé 122, ID 61) Je prends autant de cours en français, mais certains ne sont pas offerts (surtout en informatique). (énoncé 128, ID 66)

Je suis un étudiant francophone. Par contre, certain [s] cours du côté francophone sont limités. Sur 10 cours donc 30 crédit [s] le sont en anglais. (énoncé 155, ID 139)

I am trying to achieve a bilingual diploma and therefore, am taking the majority of my courses in French. But due to lack 
of choice, I am forced to take on certain English courses ${ }^{50}$. (énoncé 266, ID 133)

Les étudiants qui se scolarisent « uniquement en français » sont inscrits à des programmes entièrement de langue française, tandis que ceux qui étudient « plutôt en français » ne le sont pas nécessairement ou se heurtent à un manque de cours en langue française, qu'il s'agisse de cours obligatoires à leur formation ou de cours facultatifs. En fait, les répondants qui étudient uniquement en français sont plus nombreux à être inscrits à un programme professionnel, notamment en sciences de l'éducation, que dans un programme en sciences humaines (49\% contre $41 \%$ ). Près des trois quarts $(70 \%)$ des répondants qui étudient plutôt en français sont inscrits à un programme en sciences humaines. Les cours ou les disciplines où ils disent avoir été contraints d'étudier en anglais sont souvent les mêmes, à savoir l'informatique, la biologie, la géographie, l'astronomie, l'éducation physique et les sciences politiques.

\section{c) Francophones qui étudient autant en français qu'en anglais}

Bien que les répondants de langue maternelle française qui ont déclaré étudier autant dans une langue que dans l'autre ne soient pas nombreux (7,6\%), tous précisent le faire essentiellement en raison du manque de cours en langue française.

Plusieurs cours sont offerts seulement en anglais. De plus, il y avait certains conflits d'horaire, donc j'ai choisi la langue qui avait un cours à l'heure convenable. (énoncé 8 , ID 83)

Mon programme est seulement offert en anglais, mais j'essai [e] de prendre le plus de cours possible en français. (énoncé 83, ID 23)

Il y a un manque de classe [s] francophone [s], donc, je doi [s] prendre un nombre équilibré de cours en français et en anglais. C'est dommage. (énoncé 106, ID 46)

50. Certains étudiants francophones ont rempli le questionnaire en anglais puisqu'ils étaient dans une classe de langue anglaise. 
Parce que quand j'ai fini l'école secondaire je suis allée au Durham Collège parce qu'il y avait seulement [un] programme en anglais. À l'université, le programme d'études anciennes est seulement offert en anglais et je ne peu[x] même pas faire une concentration complète ici. (énoncé 115, ID 54)

\section{d) Francophones qui étudient en anglais}

Qu'en est-il des étudiants qui s'instruisent essentiellement en anglais ? Chez les quinze étudiants francophones qui ont indiqué étudier plutôt en anglais ou uniquement en anglais, la majorité allègue que le choix découle du fait que le programme ou les cours ne sont pas disponibles en français ${ }^{51}$ ou du fait qu'il y a une plus grande gamme de cours de langue anglaise.

J'ai choisi de faire mes études en anglais parce qu'il y a pas de cours en français pour la maîtrise que je veux faire. (énoncé 110, ID 49)

I started my program in French but as the years go on fewer and fewer of my courses are offered in French. (énoncé 261, ID 131)

Mon programme est seulement en anglais dans la $2^{e}, 3^{e}$ année supérieur [e] (pour les cours de physique). (énoncé 22, ID 93)

Only offered in English. (énoncé 168, ID 143)

I study in English because there are more classes offered than in French. There is more choice. (énoncé 292, ID 135)

Ces étudiants font aussi valoir une meilleure compétence en anglais et un plus grand usage de la langue anglaise.

With my father and boyfriend being English, I've learnt to communicate more in English. (énoncé 159, ID 141)

Ifeel that I can express myself better in English than in French. Both for speaking and writing. I didn't speak French for a long time and felt that I « lost» some of my French - Terminology is very much different in English and French - Better to stay in English because went to English high school. (énoncé 166, ID 142)

51. Plus de la moitié de ceux-ci (55\%) sont, dans l'un et l'autre des cas, inscrits à des programmes en sciences humaines. 
Ifeel as though I comprehend the material in English more, so [I] can express myself better in English (in written). (énoncé 179, ID 146)

Chez ces étudiants, le passage à l'anglais comme langue d'instruction s'était souvent opéré antérieurement. En fait, ils représentent la majorité de ceux qui, à un moment ou à un autre de leur parcours scolaire, sont passés à l'anglais comme langue principale d'enseignement. Chez ceux qui ont dit étudier à l'université « plutôt » en anglais, ce transfert se manifeste surtout en $7^{\mathrm{e}}$ année : sur les 18 répondants (90\%) qui ont commencé l'école essentiellement en français, ils ne seront que 11 (55\%) à terminer le secondaire ( $12^{\mathrm{e}}$ année) essentiellement en français, alors que, dans l'ensemble de l'échantillon, ils étaient plus des trois quarts $(77,6 \%)$ à le faire. Chez les étudiants qui ont choisi d'étudier « uniquement » en anglais à l'université, le passage à l'anglais est plus marquant : seul le quart (25\%) des étudiants qui a commencé l'école élémentaire en français terminera dans cette langue. Il semble qu'on ne puisse plus dire que, pour ces répondants, le choix de la langue d'instruction à l'université relève d'une décision propre aux études postsecondaires, puisque, derechef, la décision d'étudier en anglais est antérieure à celle de poursuivre des études, bien qu'il soit impossible de savoir si la décision a été motivée, du moins en partie, par l'intention ultérieure de faire des études postsecondaires.

\section{e) Étudiants anglophones}

Notre échantillon comprenait aussi 60 locuteurs natifs de l'anglais à avoir fait une partie ou l'entièreté de leurs études antérieures en français. Parmi ces étudiants, ceux qui le font « uniquement » en français $(16,7 \%)$ expliquent leur décision par des impératifs socioprofessionnels : ils désirent enseigner dans les écoles de langue française.

J'ai choisi le programme d'éducation puisque je veux enseign[er] un jour aux jeunes francophones. J'aime beaucoup ma langue française. (énoncé 14, ID 166) 
Je veux devenir enseignante francophone, alors j'étudie uniquement en français. (énoncé 36, ID 168)

Le discours des anglophones qui disent étudier " plutôt » en français $(13,3 \%)$ et celui de ceux qui disent le faire « autant en français qu'en anglais » $(10 \%)$ n'exprime pas pourquoi il en est ainsi. Tout au plus, notons-nous que les derniers sont souvent inscrits à des programmes de langues modernes (espagnol et italien) ou de français langue seconde, d'où les différentes langues d'instruction.

I am majoring in French, therefore many of my courses are in French. They all count as language courses I think, but most of my courses are French courses. I also take at least two courses in English, however, one is always a language course. (énoncé 189, ID 204)

French is one of my specialisations, as I am taking French and English courses, in FREN program. (énoncé 198, ID 207)

Plus de la moitié des étudiants anglophones (60\%) font leurs études universitaires essentiellement dans leur langue maternelle. Tout comme leurs confrères francophones, ils allèguent une plus grande compétence langagière dans leur langue première.

Well, now that I have been studying in English since grade 10, I find it harder to study in French. (énoncé 146, ID 194)

Did not feel my French was good enough to get good grades in post-secondary level. (énoncé 160, ID 199)

Although I do want to keep my French, I don't really have the initiative or patience to take my studies in another language. (énoncé 165, ID 200)

I am in the English language program because it is my primary language. (énoncé 204, ID 208)

I find it easier to communicate, both orally and written, in English. (énoncé 248, ID 181)

Mainly because English is my $1^{\text {st }}$ language. Secondly because, due to and since my switch from French primary school to English high school, I have lost a lot of my French speaking abilities. (énoncé 272, ID 187) 
I'm studying in English because I'm more comfortable writing in English than French. (énoncé 288, ID 190)

Quelques-uns expriment néanmoins le désir de maintenir leurs acquis en langue seconde en suivant des cours.

I have taken a couple of French courses in order to improve and not lose it completely. (énoncé 264, ID 185)

Most of my history courses are in English, but I wanted to keep my French up and eventually go to French teacher's college. French gives more career options! (énoncé 239, ID 178)

Nous le rappelons : ces réponses ne peuvent pas être généralisées. Nous tenions à les présenter puisqu'elles suscitent des réflexions intéressantes sur lesquelles il y a lieu de se pencher. Dans quelle mesure les ayants droit non francophones dans les écoles de langue française de l'Ontario ainsi que les élèves dans les programmes d'immersion française - tous deux en nombre croissant $-\mathrm{s}$ 'orienteront-ils vers des programmes postsecondaires de langue française ? S'ils le font, vers quels programmes se tourneront-ils et à quelle fréquence le feront-ils ? Il y a lieu d'enquêter davantage pour voir si, à l'instar des répondants de cette étude, ils choisissent d'étudier en français, leur langue seconde, dans des créneaux professionnels spécifiques (tels que l'éducation) ou s'ils ne cherchent qu'à suivre des cours ponctuels en français pour maintenir leurs acquis linguistiques.

\section{IV - QUE SE DÉGAGE-T-IL DE LEUR DISCOURS ?}

Nous avions précédemment fait état de la complexité des facteurs qui incitaient les francophones à faire des études postsecondaires en français. Cette complexité résidait dans le fait que les motifs relevaient à la fois de l'utilitaire et du culturel, de l'institutionnel et de l'individuel. Leurs discours le corroborent. Toutefois, entre les deux facteurs les plus souvent attestés dans les études - la proximité géographique des établissements postsecondaires où il y a des programmes en français et le sentiment de compétence en français -, les étudiants de notre échantillon ne soulèvent que le dernier. Nombreux sont ceux - tant de langue française que de 
langue anglaise - qui justifient leur choix de la langue d'instruction en fonction de leur plus grande habileté à communiquer dans une langue donnée (compréhension et production orales, compréhension et production écrites). Les répondants francophones qui avaient été scolarisés essentiellement en français étaient les plus enclins à mettre de l'avant la compétence linguistique et discursive en français comme justification du choix de cette langue. À ce facteur, se rattachent souvent des motivations socioaffectives, quelquefois empreintes de propos qui illustrent leur conscience sociopolitique du fait minoritaire. Ils portent ainsi un jugement sur la vitalité de la langue et sur eux-mêmes en tant que locuteurs de cette langue.

La proximité géographique de l'Université Laurentienne, comme motif pour étudier en français, n'est pas soulevée par les répondants. Nous savons néanmoins que plus de la moitié des répondants $(51,4 \%)$ habitaient, avant de fréquenter l'université, la région du Grand Sudbury et que 7,2\% résidaient à moins de 75 kilomètres de la ville du Grand Sudbury. Les autres répondants, outre ceux du Centre-Sud (7,7\%) et ceux de l'Est ontarien $(5,2 \%)$, venaient de communautés situées dans le Nord ontarien (Chapleau, Cochrane, Haileybury, Kapuskasing, North Bay, Timmins, entre autres). Ainsi, bien que nous ne puissions pas exclure la possibilité de la proximité institutionnelle comme facteur facilitant les études postsecondaires en français, nous ne pouvons pas la confirmer, puisque le questionnaire ne permettait pas de cerner cette question.

Les motivations socioprofessionnelles ne sont pas négligeables dans le choix de la langue d'instruction : c'est ce que l'on voit chez ceux qui se destinent à l'enseignement dans les écoles franco-ontariennes. Mais il s'agit là d'un cas exceptionnel.

L'analyse met en lumière des étudiants qui poursuivent entièrement ou en partie leur formation universitaire dans une langue autre que celle de leur choix. Chez les francophones ainsi que chez les anglophones qui désirent maintenir leur langue seconde, cela signifie qu'ils sont contraints de suivre davantage de cours 
en anglais. Leurs énoncés sont éloquents à faire état du fait que les cours obligatoires ou facultatifs ne sont qu'en anglais, que le programme n'est que partiellement en français, voire entièrement en anglais : « il n'y a pas grand choix de cours en français donc...; j'aurais voulu mais... ; j'ai dû prendre des cours en anglais... ; certains cours du côté francophone sont limités... ; I am forced to take on certain English courses... ; seulement offert en anglais... ; manque de classe francophone, donc, je dois... ; C'est dommage... ; il y a pas de cours en français...; fewer and fewer of my courses are offered in French... ; There is more choice [in English]... ». Cette absence de cours et de programmes de langue française (notamment en technologie et en sciences) ne relève pas d'un choix individuel, mais d'une contrainte institutionnelle.

Nous l'avons vu, les acquis sociopolitiques des dernières décennies en matière d'éducation en langue française s'inscrivent dans un milieu fortement diglossique. Les études le montrent bien : les francophones sont de plus en plus nombreux au pays, et en Ontario, à faire des études postsecondaires. Cependant, même ceux qui ne contestent plus le bien-fondé de fréquenter une école de langue française et d'étudier dans cette langue se heurtent encore à des obstacles institutionnels lorsqu'ils arrivent à l'université.

\section{Conclusion}

Notre but était de mieux comprendre les facteurs en jeu dans la décision que prenaient des Franco-Ontariens de faire leurs études postsecondaires en français ou de les faire en anglais. Dicté par des facteurs utilitaires (disponibilité de programmes d'études et de cours) et socioculturels (représentation linguistique personnelle et collective), le choix de la langue de la scolarité pour les étudiants vivant en milieu minoritaire est, comme nous avons tenté de le démontrer, plus complexe qu'il n'y paraît de prime abord. Certes, les facteurs tels que la langue première, la langue de la scolarité antérieure et la proximité des établissements postsecondaires augmentent les possibilités qu'un jeune Franco-Ontarien fasse 
ses études universitaires ou collégiales en français. Des facteurs de 1'ordre des représentations collectives agissent aussi sur la décision d'étudier dans une langue ou dans l'autre. Mais tous ces facteurs sont insuffisants si l'on ne tient pas compte des contraintes qui découlent des institutions elles-mêmes et qui peuvent entraver la volonté de se scolariser en français. Ce sont d'ailleurs ces contraintes que souligne le Rapport du Sommet provincial des États généraux sur le postsecondaire en Ontario français paru en 2015 et mis de l'avant par le Regroupement étudiant francoontarien, l'Assemblée de la francophonie de l'Ontario et la Fédération de la jeunesse franco-ontarienne ${ }^{52}$. La complexité de la question réside ainsi dans l'influence réciproque de tous ces facteurs, qu'ils soient individuels ou collectifs, qu'ils touchent la personne ou l'institution, qu'ils confrontent le subjectif et l'objectif, et qu'ils contribuent, ce faisant, à une base possible de la revendication, du défaitisme ou du laisser-aller.

Les recherches sur la fréquentation du postsecondaire par les francophones minoritaires de l'Ontario et sur la langue de leur scolarité fournissent des pistes qui permettent de comprendre le parcours accompli, mais aussi le chemin qu'il reste à faire avant d'être en mesure de dire que le choix est réel et qu'il est possible, en Ontario, de se scolariser en français, quel que soit le programme d'études. Plusieurs questions demeurent sans réponse et des enquêtes à plus grande échelle s'imposent, notamment auprès des étudiants francophones qui s'inscrivent dans les universités de langue anglaise et auprès des étudiants de français langue seconde.

Si les études supérieures sont la force motrice de l'économie, elles sont aussi un élément incontournable de l'apport de la communauté franco-ontarienne à cette économie et à sa vitalité ethnolinguistique. En ce sens, les études postsecondaires rehaussent le niveau d'instruction des Franco-Ontariens avec tout ce que cela a de retombées sociales, notamment en termes de

52. États généraux sur le postsecondaire en Ontario français, Rapport du Sommet provincial des États généraux sur le postsecondaire en Ontario français, Regroupement étudiant franco-ontarien, Assemblée de la francophonie de l'Ontario et Fédération de la jeunesse franco-ontarienne, 10 février 2015, 41 p. 
sentiment d'appartenance (et identitaire) à la collectivité. Comme l'ont d'ailleurs montré à maintes reprises Réal Allard, Rodrigue Landry et Kenneth Deveau ${ }^{53}$, la vitalité ethnolinguistique des communautés minoritaires est tributaire d'un vécu langagier positif dans leur langue.

53. Voir, entre autres, Kenneth Deveau, Rodrigue Landry et Réal Allard, «Facteurs reliés au positionnement envers la langue de scolarisation en milieu minoritaire francophone : le cas des ayants droit de la Nouvelle-Écosse (Canada) », Revue des sciences de l'éducation, vol. 32, n 2, 2006, p. 417-437, www.erudit.org (consulté le 17 novembre 2014). 